\title{
An Example Demonstrating the Potential for Asteroseismology of DB White Dwarf Stars
}

\author{
P.A. Bradley ${ }^{1}$ and M.A. Wood ${ }^{2}$ \\ ${ }^{1}$ McDonald Observatory and Department of Astronomy, University of Texas \\ ${ }^{2}$ Department of Physics and Space Sciences, Florida Institute of Technology
}

\begin{abstract}
We present the results of a parametric survey of evolutionary models of compositionally stratified white dwarfs with helium surface layers (DB white dwarfs). Because white dwarfs are the most common final end state of stellar evolution, determining their internal structure will offer us many clues about stellar evolution, the physics of matter under extreme conditions, plus the history of star formation and age of the local Galactic disk. As a first step towards determining the internal structure of DB white dwarf stars, we provide a comprehensive set of theoretical $g$-mode pulsation periods for comparison to observations.

Because DB white dwarfs have a layered structure consisting of a helium layer overlying the carbon/oxygen core, some modes will have the same wavelength as the thickness of the helium layer, allowing a resonance to form. This resonance is called mode trapping (see Brassard et al. 1992 and references therein) and has directly observable consequences, because modes at or near the resonance have eigenfunctions and pulsation periods that are similar to each other. This results in much smaller period spacings between consecutive overtone modes of the same spherical harmonic index $\ell$ than the uniform period spacings seen between non-trapped modes. We demonstrate with an example how one can use the distribution of pulsation periods to determine the total stellar mass, the mass of the helium surface layer, and the extent of the helium/carbon and carbon/oxygen transition zones. With these tools, we have the prospect of being able to determine the structure of the observed DBV white dwarfs, once the requisite observations become available.

We are grateful to C.J. Hansen, S.D. Kawaler, R.E. Nather, and D.E. Winget for their encouragement and many discussions. This research was supported by the National Science Foundation under grants 85-52457 and 90-14655 through the University of Texas and McDonald Observatory.
\end{abstract}

\section{References:}

Brassard, P., Fontaine, G., Wesemael, F. \& Hansen, C.J. 1992, ApJS, 80, 369 